\title{
Desarrollo de la competencia intercultural en la enseñanza de inglés como segunda lengua extranjera para Traducción e Interpretación: una propuesta metodológica
}

\section{Developing Intercultural Competence in ESL for Translation and Interpreting Studies: a Didactic Approach}

\section{Laura GONZÁLEZ FERNÁNDEZ}

Universidad de Salamanca

laurago@usal.es

Recibido: julio 2018. Revisado: septiembre 2018. Aceptado: octubre 2018.

Resumen: Este trabajo busca aportar nuevas perspectivas teóricas y un modelo práctico innovador para el desarrollo de la competencia intercultural de los estudiantes de Traducción e Interpretación en el ámbito de la enseñanza de lenguas. Para ello, analiza desde una perspectiva diacrónica la evolución del estudio de la dimensión intercultural en la enseñanza de lengua extranjera, con especial atención a la impartida en los estudios de traducción. Seguidamente se analizan los hallazgos en torno a la metodología de enseñanza de lenguas que han demostrado ser más exitosos para abordar el perfeccionamiento de la comunicación intercultural. Tomándolos como base, se propone un modelo de actividades para la enseñanza de inglés como segunda 
lengua extranjera que busca mejorar la competencia intercultural del estudiante de Traducción e Interpretación como futuro mediador intercultural, poniendo especial énfasis en el desarrollo de la dimensión simbólica.

Palabras clave: competencia intercultural; competencia simbólica; enseñanza de inglés; segunda lengua extranjera; metodología; Traducción e Interpretación.

\begin{abstract}
This paper presents new theoretical perspectives and an innovative practical approach in the field of foreign languages for the development of the intercultural competence of Translation and Interpreting students. In order to do so, it carries out a diachronic study of the intercultural dimension in the field of FLT, paying particular attention to ELT in Translation and Interpreting studies. It then explores the current methodological trends in ELT that have proved to be more successful in addressing the development of intercultural communication. Taking these findings into account, it proposes a model of activities for the teaching of English as a second foreign language that seeks to improve the intercultural competence, especially its symbolic dimension, of the student of Translation and Interpreting, as a future cross-cultural mediator.
\end{abstract}

Key words: Intercultural competence; symbolic competence; English language teaching; second foreign language; methodology; Translation and Interpreting.

\title{
1. INTRODUCCIÓN
}

Desde los años noventa, con el giro cultural, numerosos especialistas en el área de los estudios de traducción han contribuido al debate sobre el papel del traductor como mediador entre culturas (Hatim y Mason 1990: 223; Toury 1995: 54; Álvarez y Vidal 1996: 5; Quale 2003: 154; Federici 2006: 31; Wolf 2008: 14-5; Bassnett 2011: 8-9; Katan 2013: 89; Wolf 2014: 180; Jiménez 2018: 177). El desarrollo de la competencia intercultural es clave durante la formación del estudiante de Traducción e Interpretación, para que pueda alcanzar los conocimientos, actitudes y habilidades del «hablante intercultural» (intercultural speaker) que definían Byram et al. en 2001 (5).

Este trabajo busca aportar nuevas perspectivas teóricas y un modelo práctico innovador para el desarrollo de la competencia intercultural de los estudiantes de Traducción e Interpretación en el ámbito de la enseñanza de lenguas. Para ello, analiza desde una perspectiva diacrónica la evolución del estudio de la dimensión intercultural en la enseñanza de lengua extranjera, con especial atención a la impartida en los estudios de traducción. Seguidamente se analizan los hallazgos en torno a la metodología de enseñanza de lenguas que han demostrado ser más exitosos para abordar el perfeccionamiento de la comunicación intercultural. Tomándolos como base, se propone un modelo de actividades para la enseñanza de inglés como segunda lengua extranjera que busca mejorar la competencia intercultural del estudiante de Traducción

Laura GONZÁLEZ FERNÁNDEZ

Desarrollo de la competencia intercultural en la enseñanza de inglés como segunda lengua extranjera para Traducción e Interpretación: una propuesta metodológica
CLINA

vol. 4-2, December 2018, 83-102

elSSN: 2444-1961

Ediciones Universidad de Salamanca - CC BY-NC-ND 
e Interpretación como futuro mediador intercultural, poniendo especial énfasis en el desarrollo de la dimensión simbólica.

\section{PASADO Y PRESENTE DE LA COMPETENCIA INTERCULTURAL DESDE LA PERSPECTIVA DE ENSEÑANZA DE LENGUAS Y TRADUCCIÓN}

Desde los años ochenta, expertos en enseñanza de lenguas extranjeras, tanto en Europa como en Estados Unidos, advierten sobre la necesidad de plantear modelos de enseñanza que partan de la integración entre la esfera lingüística y cultural (Byram 1988, 17; Celce-Murcia et al. 1995, passim; Celce-Murcia 2007, 51). Aunque no han faltado estudios teóricos sobre ambas dimensiones, parece evidente que en Europa la enseñanza de lenguas se ha caracterizado históricamente por favorecer modelos prácticos que primaban la dimensión lingüística en detrimento de la formación intercultural (Byram et al. 2001: 1; Byram 2014: 1-2). Con el fin de comprender la situación actual realizamos un breve recorrido histórico partiendo del último modelo planteado desde una perspectiva puramente lingüística.

En Aspects of the Theory of Syntax, y desde la esfera de la gramática generativa transformacional, Chomsky presenta en 1965 un modelo de adquisición de lenguas basado en la competencia lingüística que poseería un hablante/oyente ideal: «A grammar of a language purports to be a description of the ideal speaker-hearer's intrinsic competence» (4). Como reacción a esa teoría, centrada principalmente en la competencia gramatical, Hymes realiza una propuesta alternativa que toma en consideración no solo el componente lingüístico, sino también el sociocultural (1972, 271). Sin embargo, Hymes concibe esa esfera sociocultural en relación con posibles situaciones de interacción social a las que pudiera tener que enfrentarse el aprendiz, y no con la problemática derivada del contacto entre dos culturas diferentes: «... one can see that what to grammar is imperfect, or unaccounted for, may be the artful accomplishment of a social act» (1972, 272).

Canale y Swain presentan en 1980 su teoría de la competencia comunicativa para la enseñanza de lenguas extranjeras. Su modelo se aleja aún más del enfoque exclusivamente lingüístico-gramatical, para abrirlo al comunicativo (2), y enumeran como componentes de importancia equivalente las competencias gramatical, sociolingüística y estratégica (27). Al describir su teoría parecen apuntar hacia la importancia de las culturas en contacto, pero sin llegar a articular una hipótesis clara en ese sentido: "We assume that a theory of communicative competence interacts (in as yet unspecified ways) with a theory of human action and with other systems of human knowledge (e.g. world knowledge)» (29). La competencia intercultural permanece ausente de los estudios teóricos hasta que Byram en Europa (1988) y Celce-Murcia et al. en Estados 
Unidos (1995) proponen integrar el trabajo y desarrollo de la competencia intercultural en la enseñanza de lenguas.

Este nuevo enfoque del aprendizaje de lenguas busca aportar un crecimiento integral al individuo, incorporando la dimensión intercultural a las competencias trabajadas en modelos anteriores. Beacco y Byram definen la competencia intercultural como una "combination of knowledge, skills, attitudes and behaviours which allow a speaker, to varying degrees, to recognise, understand, interpret and accept other ways of living and thinking beyond his or her home culture. This competence is the basis of understanding among people, and is not limited to language ability» $(2007,114)$. El nuevo modelo de aprendizaje buscaría desarrollar, junto al resto de saberes, habilidades y competencias implicados tradicionalmente en el aprendizaje de lenguas, la capacidad del individuo para reflexionar sobre las múltiples facetas implicadas en la identidad del individuo, y así conocer mejor al otro y conocerse mejor a sí mismo: «it is through a knowledge of others and their otherness (a kind of «detour» approach to learning) that a full awareness is acquired of the different facets of one's own identity» (Cavalli 2009, 11).

Sin embargo, parece que no resulta sencillo implementar el nuevo enfoque en el aula. En la revisión del modelo que había presentado una década antes (Celce-Murcia et al. 1995), publicada en 2007, Celce-Murcia se hace eco, a propósito de la enseñanza de lenguas en EEUU, del mismo obstáculo que Byram identifica ya en 2001 en relación con las distintas metodologías de enseñanza de lenguas en Europa, un problema que en la actualidad continúa sin resolverse de manera enteramente satisfactoria: «...second and foreign language teachers typically have far greater awareness and knowledge of linguistic rules than they do of sociocultural behaviours and expectations that accompany use of the target language» (Celce-Murcia 2007, 46). Tanto CelceMurcia (2007) como Byram (2014) lamentan que los avances realizados desde el punto de vista teórico en cuanto a la integración del aspecto cultural y lingüístico, aún no se han materializado de forma plausible y generalizada en las aulas. Byram apunta una posible razón: «The demands on teachers are often such that they will not devote what is seen to be extra energy to a cultural dimension, which itself suggests that the integration of culture and language is not yet understood» (2014).

Se hace necesario, por tanto, redirigir el foco de atención de los nuevos modelos de enseñanza de lengua extranjera para tratar de materializar la integración eficaz entre el desarrollo de las competencias lingüística y cultural del alumno. El Consejo de Europa advierte de esta necesidad e insta a los expertos en el área a trabajar en esa línea en el documento A Handbook for Curriculum Development and Teacher Training:

For plurilingual and intercultural education, it is vital to expose learners, in clearly identified teaching sequences, to many forms of writing. The experience of reading, reformulating and producing these forms is essential to familiarise learners with the

LaURa GONZÁLEZ FERNÁNDEZ

Desarrollo de la competencia intercultural en la enseñanza de inglés como segunda lengua extranjera para Traducción e Interpretación: una propuesta metodológica
CLINA

vol. 4-2, December 2018, 83-102

elSSN: 2444-1961

Ediciones Universidad de Salamanca - CC BY-NC-ND 
multitude of academic and educational cultures of contemporary societies, and with the many verbal forms given to knowledge (Beacco 2016a, 38).

Si las razones para propugnar este cambio son evidentes en el ámbito de la enseñanza de lengua extranjera en general, son aún más acuciantes en los estudios de Traducción e Interpretación, donde la educación intercultural debe ser uno de los objetivos fundamentales. Es necesario desarrollar estrategias didácticas y materiales pedagógicos dirigidos a fomentar «open, reflective and critical attitudes in order to learn to take a positive view of ... all forms of contact with otherness ... to mitigate the ego-/ethnocentric attitudes which arise from encounters with the unknown" (Beacco 2016b, 12).

Desde Estados Unidos, Kramsch ofrece su diagnóstico y apunta una posible solución que podría ser clave para superar los obstáculos planteados, en una línea de investigación no suficientemente explorada en Europa. Según Kramsch, el enfoque comunicativo ha primado eficacia y rapidez en la resolución de tareas sobre la necesidad de comprender tanto al otro como a uno mismo, necesidad que es base fundamental de la competencia intercultural (2006, 250-51). Esto ha afectado de forma especialmente negativa al estudio de lenguas en la etapa universitaria:

These developments have direct consequences for how we teach languages at the collegiate level. It is no longer appropriate to give students tourist-like competence to exchange information with native speakers of national languages within well-defined national cultures. They need a much more sophisticated competence in the manipulation of symbolic systems (Kramsch 2006, 251).

Según la autora, un individuo que es interculturalmente competente no solo conoce a fondo la otra cultura y la propia, y ha desarrollado capacidad de pensamiento crítico y de discernimiento entre el otro y uno mismo, sino que, además, mediante la reflexión sobre la cultura, concebida (desde un punto de vista post-estructuralista) como discurso, ha adquirido el conocimiento y el control de la representación, la acción y el poder simbólicos que implica la competencia simbólica: «...we are looking at the interculturally competent individual as a symbolic self that is constituted by symbolic systems like language, as well as by systems of thought and their symbolic power» (Kramsch 2010, 356).

Kramsch presenta el concepto de "competencia simbólica» como aquella que falta en la enseñanza de lenguas en el ámbito universitario si se pretende formar a estudiantes en la competencia intercultural:

The symbolic dimension of intercultural competence calls for an approach to research and teaching that is discourse-based, historically grounded, aesthetically

LaURa GONZÁLEZ FERNÁNDEZ

Desarrollo de la competencia intercultural en la enseñanza de inglés como segunda lengua extranjera para Traducción e Interpretación: una propuesta metodológica 
sensitive, and that takes into account the actual, the imagined and the virtual worlds in which we live (Kramsch 2011, 254).

La dimensión simbólica no se refiere a una posible función connotativa del lenguaje, como a priori se podría interpretar, sino al potencial de creación y estructuración de nuevos significados mediante el discurso, así como de interacción y transformación mutua en el hecho intercultural (2011, 364-365). En esta línea, redefine el aprendizaje de lenguas extranjeras como «... acquiring a symbolic mentality that grants as much importance to subjectivity and the historicity of experience as to the social conventions and the cultural expectations of any one stable community of speakers" (Kramsch 2011, 365).

Coincidimos con Kramsch en la necesidad de incluir la profundización en esta dimensión simbólica en la propuesta de nuevos modelos de enseñanza de lenguas para Traducción e Interpretación. El estudiante debe desarrollar la capacidad analítica, crítica y autocrítica que supone la competencia simbólica, con el fin de completar sus habilidades interculturales para poder convertirse en un futuro mediador entre culturas.

\section{AVANCES EN TORNO A LA FORMACIÓN INTERCULTURAL Y LA METODOLOGÍA DE ENSEÑANZA DE LENGUAS: APLICACIÓN A UNA NUEVA PROPUESTA METODOLÓGICA}

Los trabajos analizados en el apartado anterior, es decir, aquellos modelos pedagógicos desarrollados tras la publicación de «On Communicative Competence» de Hymes y propuestos dentro del marco de la metodología comunicativa, así como los que surgieron con posterioridad, sin duda supusieron una revolución en el ámbito de la enseñanza de lenguas con respecto a los modelos anteriores, basados principalmente en el perfeccionamiento de la competencia lingüística o gramatical. A pesar de sus posibles limitaciones, todos ellos han aportado excelentes avances que han demostrado su eficacia en relación con el aprendizaje, y que, por tanto, no tendría ningún sentido dejar de lado a la hora de diseñar nuevos modelos de actividad en el aula.

Seguidamente presentamos de manera resumida las metodologías y modelos de actividades que consideramos como hallazgos clave de los trabajos de investigación analizados en la sección previa. Añadiremos también algunos provenientes de otros ámbitos de la enseñanza, que por su naturaleza resultan de gran utilidad para reforzar la adquisición de la dimensión simbólica.

Con respecto a los modelos pedagógicos basados en la competencia comunicativa, destacamos como mayor logro el enfoque comunicativo en sí. Aunque aún adolece de tener una visión instrumental de la enseñanza de lenguas, el modelo de Canale y 
Swain consigue un gran avance en relación con el fomento de las habilidades comunicativas que el aprendiz necesita para enfrentarse a cualquier intercambio social y/o cultural (1980, 33). Es de importancia clave para el estudiante abordar de modo habitual la interacción comunicativa, competencia esencial para lograr objetivos de aprendizaje complejos y específicos, como son el análisis crítico del discurso, o la capacidad para realizar «traducción cultural», de la que hablaremos más adelante.

Consideramos que los mayores hallazgos de los modelos presentados por CelceMurcia y Byram son el carácter interactivo de las actividades de aula (Celce-Murcia 2007, 54-55; Byram 2001, 3) y la contextualización del aprendizaje de la lengua por medio del uso de materiales auténticos, que buscan profundizar en la interpretación del discurso de otros y en la elaboración del propio. Según Celce-Murcia, los materiales utilizados «... must be grounded in some type of real-world discourse: a story, a dialogue/conversation, a cartoon strip with accompanying language ... an e-mail message, a letter, a recipe, etc.» $(2007,51)$. El uso de textos auténticos de cierta complejidad, tanto de fondo como de forma, sin duda representa para el estudiante un reto en cuanto a decodificación del mensaje, a la vez que un modelo de calidad para el desarrollo de las destrezas de expresión oral y escrita.

Como señala Kramsch, el problema estriba en que este tipo de materiales responden a un diseño pedagógico aún altamente instrumental, centrado en adquirir la lengua para resolver tareas eficazmente (2006, 250), no en profundizar en el «tercer espacio» que Michaela Wolf, bajo la influencia de Homi Bhabha y los estudios etnográficos, define como «a space of interactive encounter which involves re-contextualization and the production of meaning» (Wolf 2008, 15). Creemos que un nuevo modelo debe plantear el uso de materiales más indicados para el desarrollo de la competencia simbólica. Como Kramsch, defendemos el uso de textos históricos y literarios como el mejor medio para profundizar en la comprensión del otro y de uno mismo, para cultivar ese tercer espacio de interacción cultural en una medida que resultaría imposible a través de los textos más utilitarios propuestos por metodologías anteriores.

Para lograr este objetivo de la enseñanza de lengua extranjera en el entorno universitario, es necesario trabajar con textos complejos, tanto desde el punto de vista formal como del contenido, que permitan al estudiante ahondar en el análisis del discurso del otro: «we have to understand what they remember from the past, what they imagine and project onto the future, and how they position themselves in the present. And we have to understand the same things of ourselves» $(2006,251)$. No basta, pues, con adquirir conocimientos sobre el significado objetivo de palabras y expresiones, o sobre el uso efectivo de los mismos en intercambios sociales de corte práctico. Es necesario también que el estudiante descubra «the cognitive and affective values of ... words and how they potentially channel a speaker's perceptions of social reality» (Kramsch y Hua 2016), es decir, debe cultivar su dimensión simbólica. 
Por su parte, Vinall aporta concreción a las teorías de Kramsch, y determina los tres componentes imprescindibles en el diseño metodológico en enseñanza de lenguas para explorar la competencia simbólica. Los presentamos brevemente:

1. Relationality: ... a relational pedagogy, a critical reflection on the meanings that emerge through interaction: across texts and modalities, across historical moments, across geographical boundaries...

2. Transgression: ... symbolic competence requires that learners also transgress boundaries, as they reflect on themselves, their own worldviews, and the languages they use.

3. Potentiality: ... The goal is to recognize the potential to create new meanings and to decide when and how to act on this potential (Vinall 2016, 5-6).

Por tanto, la metodología utilizada para potenciar el desarrollo de la competencia simbólica debe poner en relación textos, culturas, momentos históricos, experiencias individuales, buscar la reflexión dialogada sobre los significados que emergen a través de la pluralidad de interpretaciones, incitar al alumno a buscar nuevas perspectivas y a realizar un ejercicio de empatía e identificación con el otro. Debe también guiar al alumno para descubrir y crear nuevos significados a través del diálogo entre culturas.

En cuanto al tipo de actividades pedagógicas que se proponen, siempre desde un enfoque comunicativo, se fomenta el desarrollo de actividades de corte colaborativo, ya que el estudiante de lenguas en el ámbito de los estudios de traducción se beneficia doblemente del cultivo del diálogo y la negociación del significado del discurso. La metodología de aprendizaje colaborativo está especialmente indicada en este sentido, ya que responde al principio de que la realidad, lejos de ser unívoca y ajena a la percepción del individuo, «...is produced and understood through interchanges between people, shared objects, and activities, as individuals make and experience meaning together» (Barkley et al. 2014, 9). Por esta razón, propondremos actividades que responden a los estándares de la metodología colaborativa: diseño ad hoc con la intención deliberada de promover un aprendizaje articulado y en colaboración con el otro (interdependencia), fomento del pensamiento crítico, e impulso de relaciones interpersonales positivas. El primer paso del estudiante hacia la comunicación intercultural sería por tanto la práctica de un diálogo abierto y constructivo con sus propios compañeros. Esta experiencia de intercambio con el otro prepara al alumno de manera natural para el aprendizaje, comprensión y respeto de la otra cultura, y ahonda en su dimensión simbólica.

Como recurso pedagógico, a la vez que enfoque de la comunicación en el aula, se considera especialmente positivo el fenómeno del translanguaging, definido por García y Lin como «...using our language to reinforce the other in order to increase understanding and augment the pupil's activity in both languages» (2016, 3). La posibilidad

LaUra GONZÁLEZ FERNÁNDEZ Desarrollo de la competencia intercultural en la enseñanza de inglés como segunda lengua extranjera para Traducción e Interpretación: una propuesta metodológica 
de utilizar textos en la $L 1$ o la $L 2$ del individuo, traducir de una lengua y cultura a la otra, dialogar en un idioma sobre un texto escrito en el otro y viceversa, desarrolla la capacidad del individuo «...to act socially as bilinguals and multilinguals, to have two or more cultural and socio-political identities» (Otheguy y García 2018, 3). Además, apoya el perfeccionamiento de la competencia intercultural en su vertiente simbólica, ya que incita al alumno a cambiar constantemente de perspectiva cultural, previene conductas esencialistas y promueve actitudes empáticas. Requiere de él la búsqueda y fomento constante del tercer espacio, ahondar en el significado de los textos, en la producción del significado propio, y en el análisis del ajeno. Finalmente, este modo de enfocar la comunicación sigue, además, los dictados del Consejo de Europa (Cenoz y Gorter 2017, 312), pues está en línea con el concepto de plurilingüismo definido en el Marco común europeo de referencia para las lenguas (MCER). El uso de este recurso pedagógico no se restringiría a ciertas actividades concretas, sino que más bien se propiciaría en aquellos momentos en los que el uso de la L1 enriquezca claramente el aprendizaje y progreso de los estudiantes.

Consideramos útil la propuesta de recurrir a la «traducción cultural» formulada por Sturge $(2009,67-70)$ y Laviosa $(2018,579)$ como parte de las actividades que se propondrán. Sturge define el concepto de «traducción cultural» como «those practices of literary translation that mediate cultural difference, or try to convey extensive cultural background, or set out to represent another culture via translation» (67). Tanto el tipo de textos elegidos, como la actividad en sí se prestan especialmente para la profundización en las competencias necesarias para la mediación intercultural, a la vez que contribuyen al desarrollo de la práctica traductora del estudiante. La «traducción cultural», como un tipo de actividad en contraste con la «traducción lingüística», permite al aprendiz desarrollar «a symbolic mentality that is aware of the cross-cultural context in which language unfolds» (Laviosa 2018, 580). En nuestra opinión, la traducción cultural, al contrario que la traducción lingüística, profundizaría en el tercer nivel de cultura, el de la identidad cultural (Katan 2009, 72), que afecta a los valores éticos y trascendentales del individuo, preparando al aprendiz para el desarrollo de su papel como mediador cultural, ya que, como argumenta Laviosa, "cultural translators exercise ethical, ideological and political agency» $(2018,580)$.

\section{PROPUESTA METODOLÓGICA PARA EL DESARROLLO DE LA COMPETENCIA INTERCULTURAL CON ESPECIAL ATENCIÓN A LA DIMENSIÓN SIMBÓLICA}

La propuesta metodológica que se desarrolla a continuación se enmarca dentro del bloque de asignaturas Segunda Lengua Extranjera I: Inglés y Segunda Lengua Extranjera II: Inglés, impartidas en segundo curso en la titulación de Traducción e 
Interpretación. El objetivo es fomentar el desarrollo y consolidación de las destrezas, las competencias y los conocimientos necesarios para abordar el desarrollo de la competencia traductora en su segunda lengua de trabajo en Traducción e Interpretación. Las clases de ambas asignaturas se distribuyen en dos sesiones semanales de 110 minutos cada una. Con este bloque de asignaturas se busca que los estudiantes puedan adquirir y consolidar el nivel C1 del MCER. Como usuario competente de la lengua, el estudiante en este nivel:

Es capaz de comprender una amplia variedad de textos extensos y con cierto nivel de exigencia, así como reconocer en ellos sentidos implícitos.

Sabe expresarse de forma fluida y espontánea sin muestras muy evidentes de esfuerzo para encontrar la expresión adecuada.

Puede hacer un uso flexible y efectivo del idioma para fines sociales, académicos y profesionales.

Puede producir textos claros, bien estructurados y detallados sobre temas de cierta complejidad, mostrando un uso correcto de los mecanismos de organización, articulación y cohesión del texto (26).

Desde el punto de vista del resto de las competencias implicadas en el aprendizaje de las lenguas, el estudiante cuenta con un nivel avanzado (usuario competente), que le permitirá abordar la interacción oral con fluidez, espontaneidad y eficacia, leer textos complejos (incluidos los literarios), así como realizar traducciones al español y crear textos claros y estructurados en inglés sobre temas complejos (MCER, 30). Todo ello le permitirá profundizar en la adquisición de los conocimientos, destrezas, actitudes y conductas implicados en la competencia intercultural y la dimensión simbólica.

Autores como Olk han detectado que, desde el punto de vista de la interculturalidad, a veces el problema de falta de conocimiento o comprensión cultural no está solo asociado a la cultura meta, sino también a la cultura propia (2009). Por desgracia, esta situación es más habitual de lo que nos gustaría a los docentes de lenguas y de traducción.

Para nuestra propuesta metodológica se plantea, por tanto, el estudio de un tema de la cultura meta muy asociado, a su vez, a la cultura origen, con el fin de profundizar en el conocimiento de ambas a la par: combinamos historia y literatura, como aconseja Kramsch, para analizar las razones que llevaron a la segunda generación del movimiento modernista inglés al compromiso político y a involucrarse en la Guerra Civil española, y cómo eso modificó su modo de entender la vida y la literatura. Los textos que se utilizarán, auténticos en todos los casos, son dos ensayos sobre dicha generación y

LaURa GONZÁLEZ FERNÁNDEZ

Desarrollo de la competencia intercultural en la enseñanza de inglés como segunda lengua extranjera para Traducción e Interpretación: una propuesta metodológica 
dos poemas cortos inspirados por la experiencia de sus autores en la Guerra Civil. Los cuatro textos beben a la par de fuentes literarias e históricas y están indisolublemente unidos a las dos culturas en las que los estudiantes deben profundizar.

"The Leaning Tower» de Virginia Woolf (1940), autora perteneciente a la primera generación modernista, y «Background to the Thirties», de Stephen Spender (1978), escritor de la segunda generación modernista, son dos ensayos de 20 y 17 páginas respectivamente que ofrecen un marco socio-político-cultural del tema y los acontecimientos abordados. Además, ambos textos analizan, desde perspectivas distintas, las razones de corte ético, histórico, social y literario que llevaron a la segunda generación de escritores modernistas a embarcarse en el compromiso político, y las consecuencias que esta elección tuvo sobre su obra literaria y sobre sus propios valores y convicciones. "Regum Ultima Ratio», de Stephen Spender (1939), y «Última muerte», de Manuel Altolaguirre (1937) son dos poemas que muestran el lamento amargo de dos escritores ante las consecuencias devastadoras del conflicto español (y por ende, de cualquier conflicto armado) sobre las personas que se ven obligadas a participar en él.

De acuerdo con las recomendaciones de Kramsch y Vinall, en los textos propuestos se busca la pluralidad de voces, perspectivas y significados, la presencia de ambas culturas, origen y meta, y a la vez, la individualidad de cada uno de los emisores. Los textos plantearán a los estudiantes el reto de descubrir los distintos significados que adquiere la realidad, no solo en función de la cultura, sino también de las circunstancias específicas del escritor o el lector, del hablante o el oyente, del momento histórico, de su grado de profundización tanto en la cultura origen como en la cultura meta, de su capacidad de empatía en relación con el discurso ajeno, de su sensibilidad moral, lingüística y literaria. Las tareas encomendadas en relación con estos textos, como se ha señalado en el apartado anterior, estimulan al alumno a profundizar en el tercer espacio y desarrollar las competencias simbólica e intercultural.

\subsection{Sesión 1}

\subsubsection{Actividad cooperativa Jigsaw: lectura y comprensión en profundidad de los dos estudios}

Técnica: (metodología colaborativa) jigsaw (puzle). Esta técnica permite a los alumnos leer comprender y asimilar de forma rápida textos largos y complejos por medio del enfoque colaborativo. La clase se divide primero en «equipos base» de 4-6 personas. A cada una de ellas se le encomienda la lectura, comprensión y aprendizaje de una sección del texto con el fin de que sea capaz de explicárselo a otros. Los alumnos se reagrupan en «equipos de expertos» (personas encargadas de la misma sección del texto) para realizar esta tarea. Una vez adquiridos los nuevos conocimientos 
y preparada la exposición que llevarán a cabo para el resto de su equipo, el puzle se reorganiza, y los expertos vuelven a sus equipos base. Por orden, cada estudiante resume y explica al resto su sección del texto (Barkley et al. 2014, 212-3).

Materiales: ensayos «The Leaning Tower» (Virginia Woolf) y «Background to the Thirties» (Stephen Spender).

Temporalización: 80 minutos (35 minutos para cada texto y 20 minutos de debate).

El trabajo colaborativo mediante la técnica jigsaw ha demostrado una alta efectividad a la hora de motivar a los estudiantes para responsabilizarse de su propio aprendizaje y el de sus pares, y además permite abordar la comprensión de textos largos y complejos en períodos de tiempo breves de trabajo intenso. Propicia el diálogo fluido, la capacidad de análisis del discurso, el estudio de la pluralidad de interpretaciones a las que se presta cada texto, etc. El objetivo de esta actividad es abordar la apropiación del contenido de los textos y la reconstrucción de su significado desde una perspectiva plural, multicultural y multilingüe, en línea con las teorías sobre la construcción del tercer espacio (Wolf 2008, 14). Finalmente se comparten y debaten los conocimientos adquiridos.

\subsubsection{Traducción cultural: encargo de traducción-traducción anotada}

Técnica: traducción cultural en grupos de 2-3 personas.

Materiales: fragmento de 400 palabras de «Background to the Thirties» (Stephen Spender).

Temporalización: 30 minutos en el aula y 60-90 minutos de trabajo autónomo.

Esta actividad se inicia en la segunda parte de la sesión 1, momento en que los equipos cuentan con la ayuda del profesor para aclarar sus inquietudes y dudas. Continúa como tarea no presencial que los alumnos completarán de forma autónoma antes de la siguiente sesión y subirán a la plataforma online de aprendizaje para su puesta en común. En parejas o tríos, los estudiantes realizan una traducción anotada inglésespañol de una sección del ensayo de Spender donde las referencias a elementos culturales de la cultura británica son inusualmente abundantes. El encargo simulado determina que el texto se debe traducir para público español, no especialista, pero muy interesado en el tema de la Guerra Civil. Los equipos deben, en primer lugar, investigar 
los elementos culturales que aparecen en la sección, comprender, evaluar y debatir las razones por las que se mencionan en el texto, decidir el modo (integrado en el texto o en forma de nota) y el grado en que el elemento cultural se debe clarificar y trasladar al texto meta. Seguidamente necesitan decidir la información que aparecerá en las notas y el sistema de organización de estas, debatir y acordar los problemas de traducción, realizar la traducción y preparar la argumentación de sus decisiones para defenderlas en clase ante el resto de los compañeros. Esta actividad contribuye al desarrollo de una mentalidad simbólica por parte de los estudiantes, ya que en todo momento su principal objetivo es buscar, en colaboración con otras personas, el mejor modo de profundizar en el significado del discurso para así poder mediar entre ambas culturas.

\subsection{Sesión 2}

\subsubsection{Corrección conjunta y debate}

Temporalización: 45 minutos.

Las traducciones anotadas de los distintos equipos se ponen en común. Cada equipo presenta su traducción y argumenta sus decisiones. Seguidamente se abre un turno de debate sobre los elementos culturales trabajados y las soluciones adoptadas.

\subsubsection{Traducción cultural: traducción al español del poema de Spender y traducción al inglés del poema de Altolaguirre}

Técnica: traducción cultural en grupos de 2-3 personas.

Materiales: poemas «Regum Ultima Ratio» (4 estrofas, 24 versos en total), de Stephen Spender y «Última muerte» (Sección I: 3 estrofas, 20 versos en total), de Manuel Altolaguirre.

Temporalización: 30 minutos en el aula y 60-90 minutos de trabajo autónomo.

Como en el caso de la actividad 4.1.2, los alumnos inician la tarea en clase, la completan fuera del aula, y se pone en común en la siguiente sesión. En parejas/ tríos, traducen dos poemas inspirados por la Guerra Civil española. El poema de Stephen Spender, en inglés, está escrito desde la perspectiva del observador externo de la guerra, desde su pertenencia a una cultura ajena a ese enfrentamiento bélico. El de Altolaguirre, en español, desde el mismo núcleo del sufrimiento provocado por la 
participación en el conflicto. Cada equipo debe analizar, debatir y comprender la forma y el contenido de ambos poemas, interpretar la intención de los autores, y estudiar su uso de la lengua y los recursos literarios para transmitir su mensaje, cargado de matices que participan por igual de la cultura a la que pertenece el escritor y de su propia experiencia individual del hecho que inspira el poema. Identificarán los posibles problemas de traducción y debatirán el método para solucionarlos, para después traducir cada uno de los poemas al otro idioma, y preparar la argumentación de sus decisiones con el fin de defenderlas ante los compañeros. Como se ha expuesto en la sección anterior, este tipo de actividad trabaja el desarrollo de la mentalidad simbólica del estudiante, y por medio de ella, su competencia intercultural.

\subsection{Sesión 3}

\subsubsection{Corrección conjunta y debate}

Temporalización: 45 minutos.

Como en la sesión anterior, se van leyendo las traducciones anotadas de los distintos equipos. Cada equipo presenta y explica su traducción, argumenta sus decisiones, y se abre un turno de debate sobre las soluciones adoptadas.

\subsubsection{Producción escrita: escritura creativa. cartas al pasado, cartas al presente}

Técnica: metodología de escritura creativa. Grupos de 2-3 personas. Basada en las técnicas de escritura creativa de Mario Rinvolucri (2018), pero con modificaciones realizadas para acomodar el tipo de texto propuesto al alumnado, temática y nivel de aprendizaje de lengua implicados en la presente propuesta.

Materiales: como base, los cuatro textos trabajados en las actividades anteriores. Además, los alumnos tienen en todo momento acceso a Internet para facilitar su proceso de documentación en caso de que sea necesario.

Temporalización: 65 minutos en el aula y 60 minutos de trabajo autónomo.

Los estudiantes se dividen en dos grupos, y cada grupo, a su vez, en parejas o tríos. Cada grupo grande, desde la nueva perspectiva y conocimientos adquiridos sobre la cultura origen y la cultura meta durante las sesiones previas, recibe el encargo de escribir una carta en la L2 (inglés). Las parejas/tríos del grupo 1, imaginan ser un poeta británico 
de la generación estudiada. Escriben una carta personal a un joven español de hoy en día. Las parejas/tríos del grupo 2, desde la perspectiva del siglo XXI, y su propia nacionalidad, escriben una carta personal al joven poeta que se comprometió con la lucha en la Guerra Civil española. Cada pareja/trío estudia la información recogida hasta el momento, decide el contenido y forma de la carta, y crea su texto. En primer lugar, esta actividad requiere el procesado, análisis crítico y comprensión de toda la información previamente trabajada y debatida. Propicia la profundización en el primero de los tres componentes definidos por Vinall (5-6) y expuestos en la sección 3 de este trabajo: la «relacionalidad», es decir, el uso de una metodología que fomenta el pensamiento crítico basado en la interacción entre distintos tipos de textos, momentos históricos, nacionalidades, etc. Seguidamente, requiere del alumno que active su capacidad de «transgresión» (Vinall, 5-6), ponerse en lugar del otro, superando los límites impuestos por las diferentes lenguas, culturas, épocas y fronteras, tanto físicas como mentales. Finalmente, ofrece la posibilidad de explorar de forma creativa posibles nuevos discursos y significados a través de la distancia en tiempo y cultura («potencialidad»). El alumno trabaja, por tanto, los tres componentes que permiten la exploración de la competencia simbólica: relacionalidad, transgresión y potencialidad (Vinall 2016, 5-6). Las cartas se comienzan a escribir durante el tiempo que dura la sesión, pero los estudiantes tienen también la posibilidad de continuar el trabajo fuera del aula.

\subsection{Sesión 4}

\subsubsection{Peer editing}

Técnica: (metodología colaborativa) peer editing. Actividad que facilita el trabajo de revisión crítica y retroalimentación mutua entre iguales (Barkley et al., 307).

Materiales: las cartas escritas por los alumnos.

Temporalización: 55 minutos.

Tras escribir sus cartas en parejas o tríos y compartirlas en la plataforma de aprendizaje, de vuelta en el aula los alumnos forman parejas donde cada alumno pertenece a uno de los dos grupos grandes acordados en la sesión anterior. Se pone en marcha una última actividad de enfoque colaborativo previa a la puesta en común. Peer editing, o el trabajo de revisión entre iguales, ofrece al alumno escritor una crítica constructiva acerca de su trabajo, y le brinda nuevas ideas y perspectivas en torno a forma y contenido. En su papel de revisor, el alumno debe identificar, analizar y enjuiciar elementos clave en torno al tipo de texto y a los contenidos trabajados. Esto le permite el desarrollo de 
estrategias y habilidades de importancia clave desde el punto de vista lingüístico, sociopragmático y cultural (Barkley et al., 307). En nuestra opinión, este tipo de tarea fomenta, además, otro tipo de habilidades esenciales para el desarrollo de las competencias simbólica e intercultural, como son las capacidades de interdependencia e interacción positivas, y de trabajo en equipo.

\subsubsection{Debate final}

Temporalización: 55 minutos.

Como colofón a estas cuatro sesiones de trabajo, se celebra un debate final, y se busca llegar a una serie de conclusiones sobre el tema trabajado.

\section{DISCUSIÓN}

Esta propuesta pedagógica constituye uno de los múltiples acercamientos posibles al desarrollo de la interculturalidad y a la profundización en la dimensión simbólica para el estudiante de inglés como segunda lengua extranjera en los estudios de Traducción e Interpretación. Con ella se busca superar problemas no resueltos por modelos anteriores. En una primera etapa no pretende sustituir el uso del libro de texto u otros recursos de aula, sino complementarlos allí donde estos resulten insuficientes para profundizar en la competencia intercultural y la dimensión simbólica. Este enfoque se presenta por ahora como complemento a bloques temáticos determinados, y para apoyar el aprendizaje de ciertos elementos lingüísticos. Por poner un ejemplo, esta propuesta pedagógica puede asociarse a un bloque centrado en alguno de estos elementos: el pasado, recuerdos, literatura, conflictos armados, tiempos verbales de pasado, escritura de textos (cartas), etc. En el futuro se podría plantear el diseño completo del programa docente de la asignatura de inglés como segunda lengua extranjera en torno al tipo de textos y actividades planteados, añadiendo todos aquellos otros elementos necesarios para abordar el resto de las competencias implicadas en el aprendizaje de lenguas para los estudios de Traducción e Interpretación.

Una propuesta metodológica centrada en el desarrollo de las competencias simbólica e intercultural sin duda ha de estar ligada a textos profundamente imbuidos de la cultura que representan, pero a la vez altamente subjetivos. El uso de textos de ámbito histórico y literario permite al alumno profundizar en el conocimiento de la cultura meta y la cultura propia, pero también en la complejidad e individualidad de la experiencia humana, en un grado mucho más alto que el tipo de texto más utilitario trabajado habitualmente en la enseñanza de lenguas extranjeras. 
La combinación de los enfoques comunicativo y colaborativo resulta amena y motivadora, fomenta la reflexión del estudiante sobre su interacción con el otro, y le permite desarrollar habilidades y destrezas clave para la adquisición de las competencias simbólica e intercultural, que por su naturaleza requieren la interacción entre individuos. El enfoque colaborativo y el uso de translanguaging trabajan la interdependencia, la empatía, la comprensión de nuevos niveles de complejidad, tanto de la cultura meta como de la propia, y contribuyen a formar el pensamiento crítico y explorar el tercer espacio, pilares necesarios para el desarrollo de la competencia simbólica.

Las actividades propuestas ofrecen una contextualización temática de los elementos lingüísticos trabajados y promueven la competencia de comunicación intercultural de los alumnos. Tanto el jigsaw, como las traducciones culturales, la escritura creativa y la revisión entre iguales trabajan su sentido de la responsabilidad para con la cultura meta y la propia, para con la información y el significado de los textos abordados, y fomentan el respeto y la apreciación de la opinión del otro, ya sea compañero de clase o representante de la otra cultura.

En combinación, las actividades y el enfoque profundizan en la dimensión simbólica de los futuros mediadores interculturales. Incitan al alumno a cultivar y visitar ese tercer espacio de interacción social y cultural (Wolf 2008, 15) que le permite ponerse en el lugar del otro, y abordan los tres componentes que Vinall planteaba como imprescindibles para la exploración de la competencia simbólica: relacionalidad, transgresión y potencialidad (Vinall 2016, 5-6). Posibilitan analizar los significados del discurso y su interrelación en ambas lenguas de trabajo a través del espacio y el tiempo. Estimulan al alumno a superar sus limitaciones ideológicas y cruzar barreras entre culturas y lenguas, y alimentan su conciencia de las potencialidades del discurso, poniendo a prueba y favoreciendo el desarrollo de su sensibilidad intercultural.

Este modelo anticipa la posibilidad de diseñar programas docentes de enseñanza de lengua extranjera basados en competencias específicas en el ámbito de la enseñanza superior, como demanda el Consejo de Europa: «In order to use the limited available time profitably, it may be judicious to specify courses according to competences. Such specification, as well as the actual content of courses, will vary greatly according to the students' main subjects: the target competences will not necessarily be the same for those specialising in mathematics, biology and cinema» $(2007,98)$. Con el fin de satisfacer las necesidades del estudiante de Traducción e Interpretación, en nuestro caso se optaría por enfatizar el desarrollo de las competencias simbólica e intercultural.

\section{CONCLUSIONES}

Los modelos actuales de enseñanza de las lenguas en Europa requieren una actualización en el diseño de estrategias didácticas y materiales pedagógicos con el 
objeto de integrar de forma adecuada el desarrollo de la competencia intercultural en el aula, ya que, a pesar de los avances en este campo, la competencia lingüística todavía disfruta de un papel preponderante.

El modelo pedagógico y las actividades propuestas suponen una apuesta firme por el diseño de un currículo de enseñanza de lenguas centrado en el desarrollo de competencias específicas. En el caso concreto de los estudios de Traducción e Interpretación se plantea redirigir la programación hacia el fomento de las competencias simbólica e intercultural, que resultan de vital importancia para el profesional de la mediación transcultural.

Los enfoques comunicativo y colaborativo favorecen el diálogo y la comprensión y creación de significados a través del discurso. El uso de textos históricos y literarios, la traducción cultural, la escritura creativa y el fenómeno del translanguaging favorecen la comprensión de la cultura meta y la propia, alimentan la dimensión simbólica del estudiante, lo alientan a avanzar en su búsqueda del tercer espacio, y promueven el cultivo de la competencia intercultural.

\section{BIBLIOGRAFÍA}

\subsection{Fuentes primarias}

AltolaguiRre, Manuel. 1937. «Última Muerte». Hora de España III (marzo): 31-32.

SPENDER, Stephen. 1939. «Regum Ultima Ratio». En Poems for Spain, ed. por Stephen Spender. London: Hogarth, 43-44.

SPENDER, Stephen. 1978. «Background to the Thirties» The Thirties and After: Poetry, Politics, People, 1930's-1970's. New York: Random House, 63-76.

WoolF, Virginia. 1940. «The Leaning Tower». Folios of New Writing II: Autumn, 11-33.

\subsection{Fuentes secundarias}

ÁlvareZ, Román y Carmen-África VIDAL. 1996. Translation, Power, Subversion. Clevedon: Multilingual Matters.

BARKLEY, Elizabeth F., Claire HOWELL MAJOR y K. PATRICIA CROSS. 2014. Collaborative Learning Techniques. San Francisco: Jossey-Bass.

BASSNETT, Susan. 2011. «The Translator as Cross-Cultural Mediator». En The Oxford Handbook of Translation Studies, ed. por Kirsten Malmjær and Kevin Windle. Oxford: Oxford University Press.

BEACCO, Jean-Claude y Michael BYRAM. 2007. From Linguistic Diversity to Plurilingual Education: Guide for the Development of Language Education Policies in Europe. Strasbourg: COE. Acceso el 9 de abril de 2018 www.coe.int/lang

Laura GONZÁLEZ FERNÁNDEZ

Desarrollo de la competencia intercultural en la enseñanza de inglés como segunda lengua extranjera para Traducción e Interpretación: una propuesta metodológica 
BEACCO, Jean-Claude et al. 2016a. A Handbook for Curriculum Development and Teacher Training. Strasbourg: COE. Acceso el 2 de noviembre de 2018 https://rm.coe.int

BEACCO, Jean Claude et al. 2016b. Guide for the Development and Implementation of Curricula for Plurilingual and Intercultural Education. Strasbourg: COE. Acceso el 2 de noviembre de 2018 https://rm.coe.int

BYRAM, Michael. 1988. "Cultural Studies in Foreign Language Education». Language, Culture and Curriculum 1 (1): 15-31.

BYRAM, Michael, Adam NICHOLS y David STEVENS. 2001. «Introduction». En Developing intercultural Competence in Practice, ed. Por Michael Byram et al. New York: Multilingual Matters, 1-8.

BYRAM, Michael. 2009. «Intercultural Competence in Foreign Languages - the Intercultural Speaker and the Pedagogy of Foreign Language Education». En The Sage Handbook of Intercultural Competence, ed. por D. K. Deardorff. Los Angeles: Sage, 321-332.

BYRAM, Michael. 2014. «Twenty-five Years On -From Cultural Studies to Intercultural Citizenship». Language, Culture and Curriculum. 27 (3), 209-225. Acceso el 2 de noviembre de 2018 dro. dur.ac.uk/17180/.

CANALE, Michael y Merril SWAIN. 1980. "Theoretical Bases of Communicative Approaches to Second Language Teaching and Testing". Applied Linguistics, 1, 1-47.

CAVAlLI, Marisa, Daniel COSTE, Alexandru CRISAN y Piet-Hein VAN DE VEN. 2009. Plurilingual and Intercultural Education as a Project. Strasbourg: COE. Acceso el 9 de abril de 2018 www. coe.int/lang

CelCe-MURCIA, Marianne, Zoltan DÖRNYEI y Sarah THURRELL. 1995. «A pedagogical framework for communicative competence: A Pedagogically motivated model with content specifications». Issues in Applied Linguistics 6(2): 5-35.

CelCE-MuRCIA, Marianne. 2007. «Rethinking the Role of Communicative Competence in Language Teaching». En Intercultural Language Use and Language Learning, ed. por Eva Alcón Saler y M. ${ }^{a}$ Pilar Safont Jorda. Springer. 41-57. DOI: 10.1007/978-1-4020-5639-0.

CENOZ, Jasone y Durk GORTER. 2017. "Translanguaging as a Pedagogical Tool in Multilingual Education". En Language Awareness and Multilingualism, ed. por Jasone Cenoz, Durk Gorter y Stephen May. Cham, Switzerland: Springer, 309-321.

CHOMSKY, Noam. 1965. Aspects of the Theory of Syntax. Cambridge, Mass.: MIT Press.

CONSEJO DE EUROPA. 2007. From Linguistic Diversity to Plurilingual Education: Guide for the Development of Language Education Policies in Europe. Strasbourg: COE. Acceso el 2 de noviembre de 2018 https://rm.coe.int

FEDERICI, Eleonora. 2006. The Translator as Intercultural Mediator. Trento: UniService.

GARCÍA, Ofelia y Angel LIN. 2016. "Translanguaging in Bilingual Education». Bilingual and Multilingual Education. Springer Encyclopedia of Language and Education (3rd ed.). Cham: Springer, 1-14.

HATIM, Basil e lan MASON. 1990. Discourse and the Translator. London, New York: Routledge.

HYMES, Dell. 1972. "On Communicative Competence». En Sociolinguistics. Selected Readings, ed. por J. B. Pride y J. Holmes. Harmondsworth: Penguin, 269-293.

JIMÉNEZ JIMÉNEZ, Antonio F. 2018. Introducción a la Traducción. Inglés-español. London and New York: Routledge.

LaUra GONZÁLEZ FERNÁNDEZ

Desarrollo de la competencia intercultural en la enseñanza de inglés como segunda lengua extranjera para Traducción e Interpretación: una propuesta metodológica
CLINA

vol. 4-2, December 2018, 83-102

elSSN: 2444-1961

Ediciones Universidad de Salamanca - CC BY-NC-ND 
KATAN, David. 2009. "Culture». En Routledge Encyclopedia of Translation Studies, ed. por Mona Baker y Gabriela Saldanha. London, New York: Routledge, 70-73.

KATAN, David. 2013. «Intercultural Mediation». Handbook of Translation Studies, vol.4, ed. por Yves Gambier y Luc Van Doorslaer, 84-91.

KRAMSCH, Claire. 2006. «From Communicative Competence to Symbolic Competence». The Modern Language Journal 90, 249-252.

KRAMSCH, Claire. 2011. "The Symbolic Dimensions of the Intercultural». Language Teaching 44 (3): 354-367.

KRAMSCH, Claire y Zhu HUA. 2016). «Language, Culture and Language Teaching». En Routledge Handbook of English Language Teaching, ed. Por G. Hall. London: Routledge, 38-50. Acceso el 2 de noviembre de 2018. http://eprints.bbk.ac.uk/15690/1/Language\%20 and\%20culture\%20in\%20ELT.pdf

LAVIOSA, Sara. 2018. "Cultural Translation in Language Teaching». En The Routledge Handbook of Translation and Culture, ed. por Sue Ann Harding y Ovidi Carbonell Cortés. London: Routledge, 574-590.

MINISTERIO DE EDUCACIÓN, CULTURA Y DEPORTE. 2002. Marco común europeo de referencia para las lenguas: aprendizaje, enseñanza, evaluación. Madrid: ANAYA.

OLK, Harald Martin. 2009. «Translation, Cultural Knowledge and Intercultural Competence» Journal of Intercultural Communication 20. Acceso el 2 de noviembre de 2018 https://immi. se/intercultural/nr20/olk.htm

OTHEGUY, Ricardo y Ofelia GARCíA. 2018. "A Translanguaging View of the Linguistic System of Bilinguals». Applied Linguistics Review. Acceso el 2 de noviembre de 2018 https:// www.degruyter.com/view/j/alr.ahead-of-print/applirev-2018-0020/applirev-2018-0020. $\mathrm{xml}$ ?format=INT, 1-27.

QUALE, Per. 2003. From St. Jerome to Hypertext: Translation in Theory and Practice. Manchester: St Jerome.

RINVOLUCRI, Mario. 2018. "Creative Writing Exercises». Humanising Language Teaching. August 2018, year 20, issue 4. Acceso el 2 de noviembre de 2018. https://www.hltmag.co.uk/ aug18/creative-writing-exercises

STURGE, Kate. 2009. "Cultural Translation». En Routledge Encyclopedia of Translation Studies, ed. por Mona Baker y Gabriela Saldanha. London, New York: Routledge, 67-70.

TOURY, Gideon. 1995. «The Nature and Role of Norms in Translation». En Descriptive Translation Studies and Beyond. Amsterdam, Philadelphia: John Benjamins, 1995, 53-69.

VINALL, Kimberly. 2016. "“Got Llorona?": Teaching for the Development of Symbolic competence». L2 Journal, 8 (1). Acceso el 2 de noviembre de 2018. https://escholarship. org/uc/item/25t4h70v

WOLF, Michaela. 2008. «Interference from the Third Space? The Construction of Cultural Identity Through Translation". En New Trends in Translation and Cultural Identity, ed. por Micaela Muñoz-Calvo, Carmen Buesa-Gómez y M. ${ }^{a}$ Ángeles Ruiz-Moneva. Newcastle Upon Tyne: Cambridge Scholars Publishing, 11-20.

WOLF, Michaela. 2014 (2002). «Culture as Translation -and Beyond: Ethnographic Models of Representation in Translation Studies». En Crosscultural Transgressions: Research Models in Translation Studies II. Historical and Ideological Issues, ed. por Theo Hermans. Manchester: St. Jerome, 180-192.

Laura GONZÁLEZ FERNÁNDEZ

Desarrollo de la competencia intercultural en la enseñanza de inglés como segunda lengua extranjera para Traducción e Interpretación: una propuesta metodológica
CLINA

vol. 4-2, December 2018, 83-102

elSSN: 2444-1961

Ediciones Universidad de Salamanca - CC BY-NC-ND 